\title{
Exploring X-Ray Lines as Scotogenic Signal
}

\author{
Gaber Faisel* \\ Department of Physics and Center for Theoretical Sciences, National Taiwan University, \\ Taipei 106, Taiwan \\ Egyptian Center for Theoretical Physics, Modern University for Information and Technology, \\ Cairo, Egypt \\ E-mail: gfaisel@hep1.phys.ntu.edu.tw
}

\section{Shu-Yu Ho ${ }^{\dagger}$}

Department of Physics, California Institute of Technology

Pasadena, CA 91125, USA

E-mail: sho3ecaltech.edu

\section{Jusak Tandean}

Department of Physics and Center for Theoretical Sciences, National Taiwan University

Taipei 106, Taiwan

E-mail: jtandean@yahoo.com

The $3.5 \mathrm{keV}$ line recently detected in the X-ray spectra of certain galaxy clusters may have had a dark matter (DM) origin. We explore some of the implications of this DM interpretation of the line in the scotogenic model, where neutrinos acquire mass radiatively via one-loop interactions with DM. Assuming the line to arise from the slow decay of fermionic DM in the model, we first obtain a number of benchmark points representing the parameter space consistent with the new data and various other constraints and then make predictions on several observables in leptonic processes. They include the effective Majorana mass in neutrinoless double-beta decay, the sum of neutrino masses, and the rate of flavor-changing decay $\mu \rightarrow e \gamma$, as well as the cross sections of $e^{+} e^{-}$collisions into final states containing nonstandard particles in the model. These are testable in ongoing or future experiments and thus offer means to probe the model further.

The 11th International Workshop Dark Side of the Universe 2015

14-18 December 2015

Kyoto, Japan

\footnotetext{
* Research was supported by research grant NTU-ERP-102R7701

†Speaker.

${ }^{\ddagger}$ Research was supported by Taiwan MOE Academic Excellence Program (Grant No. 102R891505) and National Center for Theoretical Sciences.
} 
Recently two separate collaborations have independently reported the detection of a weak unidentified emission line at an energy of $\sim 3.5 \mathrm{keV}$ in the X-ray spectra of a number of astronomical objects [1]. On the other hand, there have been analyses done by other groups that questioned these findings, as reviewed in [2]. Pending a consensus on whether the signal is real or not, one can therefore adopt the position that it does. In that case, it may have been a tantalizing hint of physics beyond the standard model (SM), being compatible with the characteristics of a line attributable to the decay of a new particle $[1,2]$.

Here we present the results of a recent study [3] under a similar assumption, namely that the 3.5-keV X-ray line does exist and originates from the decay of a dark matter (DM) fermion. Specifically, this particle belongs to the scotogenic model [4], in which light neutrinos get mass radiatively via one-loop interactions with new particles, the lightest of which can act as weaklyinteracting massive particle DM. The nonstandard ingredients of the model consist of a scalar doublet, $\eta$, and 3 singlet Majorana fermions, $N_{1,2,3}$, all of which are odd under an exactly conserved $Z_{2}$ symmetry. In contrast, the SM particles are all $Z_{2}$ even. This symmetry prevents tree-level neutrino mass generation and ensures the DM stability. Here we suppose that the DM comprises nearly degenerate $N_{1}$ and $N_{2}$, with the latter being the more massive, and the slow decay $N_{2} \rightarrow N_{1} \gamma$ is responsible for the detected $\mathrm{X}$-ray line.

In this analysis, we take into account various constraints from the DM relic abundance data, neutrino oscillation measurements, and experimental limits on flavor-violating charged-lepton decays such as $\mu \rightarrow e \gamma$. From the allowed parameter space, we make predictions on several observables. One of them is the effective Majorana mass that can be probed in ongoing and planned searches for neutrinoless double-beta decay. Another interesting quantity is the sum of neutrino masses that can be compared with numbers inferred from upcoming cosmological measurements with improved precision. Also pertinent are the rates of the loop-induced Higgs boson decays $h \rightarrow \gamma \gamma$ and $h \rightarrow \gamma Z$, which receive scotogenic contributions and are already under investigation at the LHC. In addition, we predict the cross sections of electron-positron scattering into final states involving scotogenic particles that can be tested at next-generation $e^{+} e^{-}$colliders.

We begin by expressing the Lagrangian for the interactions of the scalar particles in the scotogenic model with one another and the gauge bosons as

$$
\mathscr{L}=\left(\mathscr{D}^{\varsigma} \Phi\right)^{\dagger} \mathscr{D}_{\varsigma} \Phi+\left(\mathscr{D}^{\varsigma} \eta\right)^{\dagger} \mathscr{D}_{\varsigma} \eta-\mathscr{V},
$$

where $\mathscr{D}_{\mathcal{S}}$ denotes the covariant derivative containing the SM gauge fields, the potential [4]

$$
\begin{aligned}
\mathscr{V}= & \mu_{1}^{2} \Phi^{\dagger} \Phi+\mu_{2}^{2} \eta^{\dagger} \eta+\frac{1}{2} \lambda_{1}\left(\Phi^{\dagger} \Phi\right)^{2}+\frac{1}{2} \lambda_{2}\left(\eta^{\dagger} \eta\right)^{2} \\
& +\lambda_{3}\left(\Phi^{\dagger} \Phi\right)\left(\eta^{\dagger} \eta\right)+\lambda_{4}\left(\Phi^{\dagger} \eta\right)\left(\eta^{\dagger} \Phi\right)+\frac{1}{2} \lambda_{5}\left[\left(\Phi^{\dagger} \eta\right)^{2}+\left(\eta^{\dagger} \Phi\right)^{2}\right]
\end{aligned}
$$

and after electroweak symmetry breaking

$$
\Phi=\left(\begin{array}{c}
0 \\
\frac{1}{\sqrt{2}}(h+v)
\end{array}\right), \quad \eta=\left(\begin{array}{c}
H^{+} \\
\frac{1}{\sqrt{2}}(\mathscr{S}+i \mathscr{P})
\end{array}\right),
$$

with $h$ being the physical Higgs boson and $v$ the vacuum expectation value (VEV) of $\Phi$. The VEV of $\eta$ is zero due to the $Z_{2}$ symmetry. The masses of the new physical scalars $\mathscr{S}, \mathscr{P}$, and $H^{ \pm}$are then given by $m_{\mathscr{S}}^{2}=m_{\mathscr{P}}^{2}+\lambda_{5} v^{2}=\mu_{2}^{2}+\frac{1}{2}\left(\lambda_{3}+\lambda_{4}+\lambda_{5}\right) v^{2}$ and $m_{H}^{2}=\mu_{2}^{2}+\frac{1}{2} \lambda_{3} v^{2}$. We make 
the usual assumption that $\lambda_{5}$ is small [5], $\left|\lambda_{5}\right| \ll\left|\lambda_{3}+\lambda_{4}\right|$, implying that $m_{\mathscr{S}}$ and $m_{\mathscr{P}}$ are nearly degenerate, $\left|m_{\mathscr{S}}^{2}-m_{\mathscr{P}}^{2}\right|=\left|\lambda_{5}\right| v^{2} \ll m_{\mathscr{S}}^{2} \simeq m_{\mathscr{P}}^{2}$. The Lagrangian for the masses and interactions of $N_{k}$ is

$$
\mathscr{L}_{N}=-\frac{1}{2} M_{k} \overline{N_{k}^{\mathrm{c}}} P_{R} N_{k}+\mathscr{Y}_{r k}\left[\bar{\ell}_{r} H^{-}-\frac{1}{\sqrt{2}} \bar{v}_{r}(\mathscr{S}-i \mathscr{P})\right] P_{R} N_{k}+\text { H.c. },
$$

where $M_{k}$ represent their masses, $k, r=1,2,3$ are summed over, the superscript c refers to charge conjugation, $P_{R}=\frac{1}{2}\left(1+\gamma_{5}\right)$, and $\ell_{1,2,3}=e, \mu, \tau$. One can form a matrix $\mathscr{Y}$ for the Yukawas $\mathscr{Y}_{r k}$,

$$
\mathscr{Y}=\left(\begin{array}{ccc}
Y_{e 1} & Y_{e 2} & Y_{e 3} \\
Y_{\mu 1} & Y_{\mu 2} & Y_{\mu 3} \\
Y_{\tau 1} & Y_{\tau 2} & Y_{\tau 3}
\end{array}\right), \quad Y_{\ell_{r} k}=\mathscr{Y}_{r k}
$$

The neutrinos gain mass radiatively through one-loop diagrams with internal $\mathscr{S}, \mathscr{P}$, and $N_{k}$. The mass eigenvalues $m_{j}$ are given by [4]

$$
\begin{gathered}
\operatorname{diag}\left(m_{1}, m_{2}, m_{3}\right)=\mathscr{U}^{\dagger} \mathscr{M}_{v} \mathscr{U}^{*}, \quad \mathscr{M}_{v}=\mathscr{Y} \operatorname{diag}\left(\Lambda_{1}, \Lambda_{2}, \Lambda_{3}\right) \mathscr{Y}^{\mathrm{T}}, \\
\Lambda_{k}=\frac{\lambda_{5} v^{2}}{16 \pi^{2} M_{k}}\left[\frac{M_{k}^{2}}{m_{0}^{2}-M_{k}^{2}}+\frac{2 M_{k}^{4} \ln \left(M_{k} / m_{0}\right)}{\left(m_{0}^{2}-M_{k}^{2}\right)^{2}}\right], \quad m_{0}^{2}=\frac{1}{2}\left(m_{\mathscr{S}}^{2}+m_{\mathscr{P}}^{2}\right),
\end{gathered}
$$

where $\mathscr{U}$ is the Pontecorvo-Maki-Nakagawa-Sakata (PMNS) unitary matrix and the formula for $\Lambda_{k}$ applies to the $m_{0} \simeq m_{\mathscr{S}} \simeq m_{\mathscr{P}}$ case. For $\mathscr{U}$, we choose the standard parametrization [6]

$$
\mathscr{U}=\tilde{u} \operatorname{diag}\left(e^{\frac{i \alpha_{1}}{2}}, e^{\frac{i \alpha_{2}}{2}}, 1\right), \quad \tilde{u}=\left(\begin{array}{ccc}
c_{12} c_{13} & s_{12} c_{13} & s_{13} e^{-i \delta} \\
-s_{12} c_{23}-c_{12} s_{23} s_{13} e^{i \delta} & c_{12} c_{23}-s_{12} s_{23} s_{13} e^{i \delta} & s_{23} c_{13} \\
s_{12} s_{23}-c_{12} c_{23} s_{13} e^{i \delta} & -c_{12} s_{23}-s_{12} c_{23} s_{13} e^{i \delta} & c_{23} c_{13}
\end{array}\right),
$$

where $\delta \in[0,2 \pi]$ and $\alpha_{1,2} \in[0,2 \pi]$ are the Dirac and Majorana $C P$-violation phases, respectively, $c_{m n}=\cos \theta_{m n} \geq 0$, and $s_{m n}=\sin \theta_{m n} \geq 0$.

The Yukawa couplings $Y_{\ell_{r} k}$ need to satisfy the relations in (6). We adopt the solutions [7]

$$
\begin{aligned}
& Y_{e 1}=\frac{-c_{12} c_{13} Y_{1}}{c_{12} c_{23} s_{13} e^{i \delta}-s_{12} s_{23}}, \quad Y_{e 2}=\frac{-s_{12} c_{13} Y_{2}}{s_{12} c_{23} s_{13} e^{i \delta}+c_{12} s_{23}}, \quad Y_{e 3}=\frac{s_{13} Y_{3}}{c_{23} c_{13} e^{i \delta}}, \\
& Y_{\mu 1}=\frac{c_{12} s_{23} s_{13} e^{i \delta}+s_{12} c_{23}}{c_{12} c_{23} s_{13} e^{i \delta}-s_{12} s_{23}} Y_{1}, \quad Y_{\mu 2}=\frac{s_{12} s_{23} s_{13} e^{i \delta}-c_{12} c_{23}}{s_{12} c_{23} s_{13} e^{i \delta}+c_{12} s_{23}} Y_{2}, \quad Y_{\mu 3}=\frac{s_{23} Y_{3}}{c_{23}},
\end{aligned}
$$

corresponding to the neutrino mass eigenvalues

$$
m_{1}=\frac{\Lambda_{1} Y_{e 1}^{2} e^{-i \alpha_{1}}}{c_{12}^{2} c_{13}^{2}}, \quad m_{2}=\frac{\Lambda_{2} Y_{e 2}^{2} e^{-i \alpha_{2}}}{s_{12}^{2} c_{13}^{2}}, \quad m_{3}=\frac{\Lambda_{3} Y_{3}^{2}}{c_{13}^{2} c_{23}^{2}} .
$$

The requirement that $m_{1,2,3}$ be real and nonnegative then implies

$$
\alpha_{1}=\arg \left(\Lambda_{1} Y_{e 1}^{2}\right), \quad \alpha_{2}=\arg \left(\Lambda_{2} Y_{e 2}^{2}\right), \quad \arg \left(\Lambda_{3} Y_{3}^{2}\right)=0 .
$$

These choices are consistent with the neutrino oscillation data [8], including $\sin \theta_{13} \neq 0$. 
Information on the values of some of the neutrino parameters above is available from various measurements. A recent fit to the global data on neutrino oscillations [8] yield

$$
\begin{array}{rlrl}
\sin ^{2} \theta_{12} & =0.308 \pm 0.017, \quad \sin ^{2} \theta_{23}=0.437_{-0.023}^{+0.033}, & \sin ^{2} \theta_{13}=0.0234_{-0.0019}^{+0.0020} \\
\delta m^{2} & =m_{2}^{2}-m_{1}^{2}=\left(7.54_{-0.22}^{+0.26}\right) \times 10^{-5} \mathrm{eV}^{2}, \quad \delta / \pi=1.39_{-0.27}^{+0.38} \\
\Delta m^{2} & =m_{3}^{2}-\frac{1}{2}\left(m_{1}^{2}+m_{2}^{2}\right)=\left(2.43_{-0.06}^{+0.06}\right) \times 10^{-3} \mathrm{eV}^{2} .
\end{array}
$$

These belong to the normal hierarchy of neutrino masses $\left(m_{1}<m_{2}<m_{3}\right)$, which is preferred by the Yukawa solutions in (9). Unlike the well-determined squared-mass differences in (12), the absolute scale of the masses is still poorly known. From various cosmological observations, one can infer $\Sigma_{k} m_{k} \lesssim 0.2 \mathrm{eV}$ [6]. As for the Majorana phases $\alpha_{1}$ and $\alpha_{2}$, there is still no empirical information available on their values.

We now enumerate the pertinent constraints on our scenario of interest. We start by recalling our assumption that $N_{1}$ is cold DM and only slightly less massive than $N_{2}$ such that $N_{2} \rightarrow N_{1} \gamma$ proceeds very slowly and is responsible for the line. Moreover, $N_{2}$ has a lifetime $\tau_{N_{2}}$ longer than the age of the Universe, $\tau_{U}$, and hence contributes to the DM density with present-day fractional abundance $f_{N_{2}}$. The near degeneracy of $N_{1,2}$ implies that $f_{N_{2}} \simeq \frac{1}{2} e^{-\tau_{U} / \tau_{N_{2}}}$, where the exponential factor accounts for the depletion of $N_{2}$ after freeze-out time. As detailed in [3], it follows that we can require

$$
9.6 \times 10^{-48}<\frac{\Gamma_{N_{2} \rightarrow N_{1} \gamma}}{M_{1}} e^{-\tau_{U} / \tau_{N_{2}}}<9.2 \times 10^{-47},
$$

where $\tau_{U}=4.36 \times 10^{17} \mathrm{~s}[6]$. This radiative decay arises from loop diagrams with internal $\ell_{k}^{ \pm}$and $H^{\mp}$ and the photon attached to either one of the charged particles. Its rate is given by $[3,9]$

$$
\Gamma_{N_{2} \rightarrow N_{1} \gamma}=\frac{\alpha E_{\gamma}^{3} M_{1}^{2}}{64 \pi^{4} m_{H}^{4}}\left[\sum_{k} \operatorname{Im}\left(\mathscr{Y}_{k 1} \mathscr{Y}_{k 2}^{*}\right) \mathscr{G}\left(\frac{M_{1}^{2}}{m_{H}^{2}}, \frac{m_{\ell_{k}}^{2}}{m_{H}^{2}}\right)\right]^{2}, \mathscr{G}(x, y)=\int_{0}^{1} \frac{d u u(u-1)}{u^{2} x-(1+x-y) u+1}
$$

where $E_{\gamma} \simeq M_{2}-M_{1} \ll M_{1}$. Thus (13) translates into restrictions on $\mathscr{Y}_{k 1, k 2}$.

For the $M_{1,2}$ values considered here, ${ }^{1}$ the $N_{2}$ lifetime $\tau_{N_{2}}=1 / \Gamma_{N_{2}}$ is dominated by the three body decay $N_{2} \rightarrow N_{1} v v$ which is mediated by the neutral scalars $\mathscr{S}$ and $\mathscr{P}$ and therefore depends also on $\mathscr{Y}_{k 1, k 2}$. We employ the amplitude and rate already derived in [7].

With both $N_{1}$ and $N_{2}$ making up the relic density, its observed value constitutes another restraint on their couplings. We impose $0.1155 \leq \Omega \hat{h}^{2} \leq 0.1241$ which is the $90 \%$ confidence level (CL) range of the data $\Omega \hat{h}^{2}=0.1198 \pm 0.0026$ [6], where $\Omega$ is the present DM density relative to its critical value and $\hat{h}$ denotes the Hubble parameter. Due to the near degeneracy and mutual interactions of $N_{1,2}$, their coannihilation becomes relevant to the calculation of the relic density [11]. In that case $\Omega$ is approximately given by $[11,12]$

$$
\Omega \hat{h}^{2}=\frac{1.07 \times 10^{9} x_{f} \mathrm{GeV}^{-1}}{\sqrt{g_{*}} m_{\mathrm{Pl}}\left[a_{\mathrm{eff}}+3\left(b_{\mathrm{eff}}-a_{\mathrm{eff}} / 4\right) / x_{f}\right]}, \quad x_{f}=\ln \frac{0.191\left(a_{\mathrm{eff}}+6 b_{\mathrm{eff}} / x_{f}\right) M_{1} m_{\mathrm{Pl}}}{\sqrt{g_{*} x_{f}}},
$$

\footnotetext{
${ }^{1}$ Their numbers in our examples lead to $\left(M_{2}-M_{1}\right) /\left(M_{1}+M_{2}\right) \lesssim 10^{-8}$. Such a tiny mass split may be explained by
} the presence of an extra symmetry, e.g. particle number conservation, which allows $N_{1}$ and $N_{2}$ to form a pseudo-Dirac fermion, but which is slightly broken by highly suppressed operators $[9,10]$. 
where $m_{\mathrm{Pl}}=1.22 \times 10^{19} \mathrm{GeV}$ is the Planck mass, $g_{*}$ is the number of relativistic degrees of freedom below the freeze-out temperature $T_{f}=M_{1} / x_{f}$, and $a_{\text {eff }}$ and $b_{\text {eff }}$ are defined by the expansion of the coannihilation rate $\sigma_{\text {eff }} v_{\text {rel }}=a_{\text {eff }}+b_{\text {eff }} v_{\text {rel }}^{2}$ in terms of the relative speed $v_{\text {rel }}$ of the annihilating particles in their center-of-mass (c.m.) frame. The leading contributions to $\sigma_{\text {eff }}$ arise from (co)annihilations into $v_{i} v_{j}$ and $\ell_{i}^{-} \ell_{j}^{+}$, which are induced at tree level by $(\mathscr{S}, \mathscr{P})$ and $H^{ \pm}$ exchanges, respectively. Neglecting the $N_{1,2}$ mass difference, we have [11]

$$
\sigma_{\text {eff }}=\frac{1}{4}\left(\sigma_{11}+2 \sigma_{12}+\sigma_{22}\right), \quad \sigma_{k l}=\sigma_{N_{k} N_{l} \rightarrow v v}+\sigma_{N_{k} N_{l} \rightarrow \ell \bar{\ell}}, \quad \sigma_{12}=\sigma_{21} .
$$

These cross sections have been computed in [7, 13] and each proceed from diagrams in the $t$ and $u$ channels because of the Majorana nature of the external neutral fermions. The size of the S-wave contribution $a_{\text {eff }}$ is at least several times that of the P-wave one $b_{\text {eff }}$ and comes mainly from $\sigma_{12}$. In numerical work, we keep in (15) both $a_{\text {eff }}$ and $b_{\text {eff }}$.

There are also constraints on $\mathscr{Y}_{j k}$ from the measurements of a number of low-energy observables. These couplings enter the neutrino masses $m_{1,2,3}$ in (10) and consequently need to be consistent with the most precise mass measurements. Thus we require

$$
30.0<\frac{\Delta m^{2}}{\delta m^{2}}<34.3
$$

based on the $90 \% \mathrm{CL}$ ranges of the data on $\delta m^{2}$ and $\Delta m^{2}$ in (12).

Other loop processes are $\mu \rightarrow e \gamma$ and the modification $\Delta a_{\mu}$ to the muon's anomalous magnetic moment $a_{\mu}$, which both involve internal $H^{ \pm}$and $N_{k}$. The predictions are given by $[5,14]$

$$
\begin{aligned}
\mathscr{B}(\mu \rightarrow e \gamma) & =\frac{3 \alpha}{64 \pi G_{\mathrm{F}}^{2} m_{H}^{4}}\left|\sum_{k} \mathscr{Y}_{1 k} \mathscr{Y}_{2 k}^{*} \mathscr{F}\left(\frac{M_{k}^{2}}{m_{H}^{2}}\right)\right|^{2}, \quad \Delta a_{\mu}=\frac{-m_{\mu}^{2}}{16 \pi^{2} m_{H}^{2}} \sum_{k}\left|\mathscr{Y}_{2 k}\right|^{2} \mathscr{F}\left(\frac{M_{k}^{2}}{m_{H}^{2}}\right), \\
\alpha & =e^{2} /(4 \pi), \quad \mathscr{F}(x)=\left(1-6 x+3 x^{2}+2 x^{3}-6 x^{2} \ln x\right) /\left[6(1-x)^{4}\right],
\end{aligned}
$$

where $G_{\mathrm{F}}$ is the Fermi constant. The data are $\mathscr{B}(\mu \rightarrow e \gamma)_{\exp }<5.7 \times 10^{-13}$ at $90 \%$ CL [6] and $a_{\mu}^{\exp }-a_{\mu}^{\mathrm{SM}}=(249 \pm 87) \times 10^{-11}[15]$, the latter implying that we can require $\left|\Delta a_{\mu}\right|<9 \times 10^{-10}$.

There are other constraints, described in [13], such as those on $\tau \rightarrow(e, \mu) \gamma$, as well as theoretical ones, which turn out to be less important for what follows. Direct searches for DM may also add to the restrictions [9], but for the examples below we find that the cross sections of $N_{1}$ scattering off nuclei can evade the strongest limits from the LUX experiment [16].

After setting $\theta_{12,23,13}$ and $\delta$ to their central values from (12), taking $E_{\gamma}=3.54 \mathrm{keV}$ based on the detected X-ray energy numbers in [1], and scanning the parameter space of the model, we obtain regions satisfying the restrictions discussed above. We illustrate this in Table 1 with different sets of the mass parameters $m_{0, H}, M_{1}=M_{2}-E_{\gamma}$, and $M_{3}$ and the Yukawa constants $Y_{1,2,3}$. It is worth noting that these results yield $\tau_{N_{2}} \simeq(1.4-17) \tau_{U}$ and $\Gamma_{N_{2} \rightarrow N_{1} v v} \simeq(12-84) \Gamma_{N_{2} \rightarrow N_{1} \gamma}$. We turn next to the resulting predictions for a number of observables.

We find that the benchmark points in Table 1 can translate into $\mathscr{B}(\mu \rightarrow e \gamma)$ values that are not very close to the experimental limit $\mathscr{B}(\mu \rightarrow e \gamma)_{\text {exp }}$. We display the numbers in the second column of Table 2. Thus they serve as predictions of the scotogenic scenario under consideration that can be tested with upcoming searches for $\mu \rightarrow e \gamma$ which will expectedly reach a sensitivity at a level of a few times $10^{-14}$ within the next five years [17]. 
Table 1: Sample values of the mass parameters $m_{0, H}, M_{1} \simeq M_{2}$, and $M_{3}$ and Yukawa constants $Y_{1,2,3}$ satisfying the constraints discussed above.

\begin{tabular}{|c|ccccccc|}
\hline Set & $\frac{m_{0}}{\mathrm{GeV}}$ & $\frac{m_{H}}{\mathrm{GeV}}$ & $\frac{M_{1}}{\mathrm{GeV}}$ & $\frac{M_{3}}{\mathrm{GeV}}$ & $Y_{1}$ & $Y_{2}$ & $Y_{3}$ \\
\hline \hline I & 340 & 395 & 180 & 235 & $0.215+0.028 i$ & $0.281+0.036 i$ & 0.419 \\
II & 420 & 440 & 318 & 415 & $0.215+0.027 i$ & $0.281+0.035 i$ & 0.431 \\
III & 605 & 600 & 350 & 470 & $0.120+0.244 i$ & $0.157+0.319 i$ & 0.535 \\
IV & 1030 & 1100 & 600 & 805 & $-0.360+0.041 i$ & $-0.471+0.053 i$ & 0.716 \\
V & 1100 & 1200 & 600 & 795 & $-0.377+0.072 i$ & $-0.493+0.093 i$ & 0.750 \\
\hline
\end{tabular}

Table 2: Predictions corresponding to the benchmark points in Table 1. The last three columns contain cross sections at $e^{+} e^{-}$c.m. energies $\sqrt{s}=1,2,3 \mathrm{TeV}$.

\begin{tabular}{|c|c|c|c|c|c|c|c|c|c|c|c|}
\hline \multirow{2}{*}{ Set } & \multirow{2}{*}{$\frac{\mathscr{B}(\mu \rightarrow e \gamma)}{10^{-13}}$} & \multirow{2}{*}{$\frac{\left\langle m_{\beta \beta}\right\rangle}{\mathrm{ev}}$} & \multirow{2}{*}{$\frac{\Sigma_{k} m_{k}}{\mathrm{eV}}$} & \multirow{2}{*}{$\frac{\alpha_{1}}{\pi}$} & \multirow{2}{*}{$\frac{\alpha_{2}}{\pi}$} & \multirow{2}{*}{$\frac{\mu_{2}}{\mathrm{GeV}}$} & \multirow{2}{*}{$\mathscr{R}_{\gamma \gamma}$} & \multirow{2}{*}{$\mathscr{R}_{\gamma Z}$} & \multicolumn{3}{|c|}{$\sigma_{e \bar{e} \rightarrow H \bar{H} \rightarrow \ell \bar{\ell}^{\prime} \not}(\mathrm{pb})$} \\
\hline & & & & & & & & & 1 & 2 & 3 \\
\hline $\mathrm{I}$ & 5.6 & 0.054 & 0.20 & -0.058 & 0.15 & $101(439)$ & $0.91(1.02)$ & $0.96(1.01)$ & 0.038 & 0.059 & 0.039 \\
\hline II & 2.7 & 0.052 & 0.19 & -0.061 & 0.15 & $110(499)$ & $0.91(1.03)$ & $0.96(1.01)$ & 0.010 & 0.038 & 0.029 \\
\hline III & 3.4 & 0.050 & 0.18 & 0.57 & 0.78 & $145(665)$ & $0.91(1.02)$ & $0.96(1.01)$ & 0 & 0.060 & 0.055 \\
\hline IV & 0.93 & 0.049 & 0.18 & -0.21 & 0.001 & $255(990)$ & $0.91(0.98)$ & $0.96(0.99)$ & 0 & 0 & 0.054 \\
\hline $\mathrm{V}$ & 0.69 & 0.052 & 0.19 & -0.26 & -0.047 & $280(1070)$ & $0.91(0.98)$ & $0.96(0.99)$ & 0 & 0 & 0.047 \\
\hline
\end{tabular}

Another important observable is the effective Majorana mass

$$
\left\langle m_{\beta \beta}\right\rangle=\left|\sum_{k} \mathscr{U}_{1 k}^{2} m_{k}\right|=\left|c_{12}^{2} c_{13}^{2} m_{1} e^{i \alpha_{1}}+s_{12}^{2} c_{13}^{2} m_{2} e^{i \alpha_{2}}+s_{13}^{2} m_{3} e^{-2 i \delta}\right|
$$

which follows from the Majorana nature of the electron neutrino and can be probed in neutrinoless double- $\beta$ decay experiments [18]. This process is of fundamental importance because it violates lepton-number conservation and thus will be evidence for new physics if detected [18]. The parameters in Table 1 lead to the predictions in the third column of Table 2. They are only a few times less than the existing experimental upper limits on $\left\langle m_{\beta \beta}\right\rangle$, the best one being $0.12 \mathrm{eV}$ [6]. Forthcoming searches within the next decade are expected to have sensitivities to $\left\langle m_{\beta \beta}\right\rangle$ down to $0.01 \mathrm{eV}$ [19].

The sum of neutrino masses, $\Sigma_{k} m_{k}$, is also predicted in Table 2. The results are compatible with the aforementioned bound from cosmological observations, $\Sigma_{k} m_{k} \lesssim 0.2 \mathrm{eV}$ [6]. Upcoming data with improved precision can be expected to check the predictions.

We include in Table 2 the corresponding values of $\alpha_{1,2}$, computed using (11). Although currently there is no experimental information on the values of these phases, they may be extractable from future measurements, especially those on $\left\langle m_{\beta \beta}\right\rangle$.

Additional windows into the nonstandard sector of the model may be the Higgs boson decays $h \rightarrow \gamma \gamma$ and $h \rightarrow \gamma Z$, which arise in the SM mainly from top-quark- and $W$-boson-loop diagrams and also receive one-loop contributions from $H^{ \pm}$. Employing the formulas given in [13] with the Higgs mass $m_{h}=125.1 \mathrm{GeV}$ [6] and selecting specific values of the parameter $\mu_{2}$ in (2), we have listed in Table 2 the resulting rate ratio

$$
\mathscr{R}_{\gamma \mathscr{V}^{0}}=\frac{\Gamma\left(h \rightarrow \gamma^{\mathscr{V}^{0}}\right)}{\Gamma\left(h \rightarrow \gamma^{V^{0}}\right)_{\mathrm{SM}}}, \quad \mathscr{V}^{0}=\gamma, Z .
$$

The two numbers on each line in the $\mathscr{R}_{\gamma{ }^{0}}$ column correspond to the two numbers on the same line in the $\mu_{2}$ column. Evidently the scotogenic effects on these two modes have a positive correlation. 
Since the branching fraction of $h \rightarrow \gamma \gamma$ has been measured, we can already compare our examples with the LHC data: $\mathscr{B}(h \rightarrow \gamma \gamma) / \mathscr{B}(h \rightarrow \gamma \gamma)_{\mathrm{SM}}=1.16_{-0.18}^{+0.20}$ [20]. Its $90 \% \mathrm{CL}$ range is compatible with the predictions, but the situation may change when more data become available.

As investigated in [7], next-generation $e^{+} e^{-}$colliders, such as the International Linear Collider [22] and the Compact Linear Collider [23], have the potential to provide extra means to check the scotogenic model further. Their c.m. energies may be as high as $3 \mathrm{TeV}$ or more [22, 23]. Here we consider the scattering $e^{+} e^{-} \rightarrow H^{+} H^{-}$followed by the (sequential) decays of $H^{ \pm}$into $\ell_{j}^{ \pm} N_{1}$ possibly plus neutrinos. Since $N_{1}$ is DM and the neutrinos are undetected, this process contributes to the channel $e^{+} e^{-} \rightarrow \ell^{+} \ell^{\prime-} \mathbb{E}^{\text {with }}$ wissing energy $\mathbb{E}$, summed over the final charged leptons. Employing the pertinent expressions derived in [7], we collect the scotogenic contributions in the last three columns of Table 2 for c.m. energies $\sqrt{s}=1,2,3 \mathrm{TeV}$, respectively. The main background is the SM scattering $e^{+} e^{-} \rightarrow W^{+} W^{-} \rightarrow v v^{\prime} \ell^{+} \ell^{\prime-}$ summed over the final leptons. Compared to the SM tree-level cross-sections $\sigma_{e \bar{e} \rightarrow W \bar{W} \rightarrow v v^{\prime} \ell \bar{\ell}^{\prime}}=0.28,0.10,0.05 \mathrm{pb}$ at $\sqrt{s}=1,2,3 \mathrm{TeV}$, respectively, the scotogenic numbers can clearly be similar in size and hence are testable at these future colliders.

In conclusion, we have explored possible implications of the unidentified $3.5 \mathrm{keV}$ line recently reported in the X-ray spectra of some astronomical objects in the context of the scotogenic model. Assuming that this finding can stand future scrutiny and that no better standard explanations are available for it, we consider the scenario in which the line originates from the decay of fermionic DM in the model. Particularly, DM is composed of nearly degenerate $N_{1}$ and $N_{2}$, the latter being slightly more massive, and the slow decay $N_{2} \rightarrow N_{1} \gamma$ is responsible for the detected X-ray line. We take into account various restraints on the model, especially those from the observed DM relic abundance, neutrino oscillation data, searches for flavor-violating lepton decays such as $\mu \rightarrow e \gamma$, and measurements of the muon $g-2$. Subsequently, we select from the allowed parameter space several benchmark points to make predictions on a number of interesting observables in processes involving leptons. These include the effective Majorana mass that can be probed in ongoing and planned searches for neutrinoless double-beta decay, the sum of neutrino masses that can be compared to numbers inferred from upcoming cosmological measurements with improved precision, the $\mu \rightarrow e \gamma$ branching-ratio that will confront further experimental checks not too long from now, and the rates of the Higgs decays $h \rightarrow \gamma \gamma$ and $h \rightarrow \gamma Z$ presently being examined at the LHC. Many of the predictions are already within reach of running or near future experiments. We also evaluate the cross section of $e^{+} e^{-} \rightarrow H^{+} H^{-} \rightarrow \ell^{+} \ell^{\prime-} \notin$ which is testable at next-generation $e^{+} e^{-}$colliders, such as the ILC. These machines can, in addition, offer a cleaner environment than the LHC to measure $h \rightarrow \gamma \gamma, \gamma Z$. Thus our analysis indicates that X-ray lines from certain astronomical objects can potentially provide extra means to scrutinize the scotogenic model.

\section{References}

[1] E. Bulbul et al., Astrophys. J. 789, 13 (2014) [arXiv:1402.2301]; A. Boyarsky, O. Ruchayskiy, D. Iakubovskyi, and J. Franse, Phys. Rev. Lett. 113, 251301 (2014) [arXiv:1402.4119]; Phys. Rev. Lett. 115, 161301 (2015) [arXiv:1408.2503].

[2] D. Iakubovskyi, arXiv:1510.00358 [astro-ph.HE].

[3] G. Faisel, S. Y. Ho and J. Tandean, Phys. Lett. B 738, 380 (2014) [arXiv:1408.5887]. 
[4] E. Ma, Phys. Rev. D 73, 077301 (2006) [hep-ph/0601225].

[5] J. Kubo, E. Ma, and D. Suematsu, Phys. Lett. B 642, 18 (2006) [hep-ph/0604114].

[6] K.A. Olive et al. [Particle Data Group Collaboration], Chin. Phys. C 38, 090001 (2014).

[7] S.Y. Ho and J. Tandean, Phys. Rev. D 89, 114025 (2014) [arXiv:1312.0931].

[8] F. Capozzi et al., Phys. Rev. D 89, 093018 (2014) [arXiv:1312.2878].

[9] D. Schmidt, T. Schwetz, and T. Toma, Phys. Rev. D 85, 073009 (2012) [arXiv:1201.0906].

[10] A. De Simone, V. Sanz, and H.P. Sato, Phys. Rev. Lett. 105, 121802 (2010) [arXiv:1004.1567].

[11] K. Griest and D. Seckel, Phys. Rev. D 43, 3191 (1991).

[12] G. Jungman, M. Kamionkowski, and K. Griest, Phys. Rept. 267, 195 (1996) [hep-ph/9506380]; J. Edsjo and P. Gondolo, Phys. Rev. D 56, 1879 (1997) [hep-ph/9704361].

[13] S.Y. Ho and J. Tandean, Phys. Rev. D 87, 095015 (2013) [arXiv:1303.5700].

[14] E. Ma and M. Raidal, Phys. Rev. Lett. 87, 011802 (2001) [Erratum-ibid. 87, 159901 (2001)] [hep-ph/0102255].

[15] T. Aoyama, M. Hayakawa, T. Kinoshita, and M. Nio, Phys. Rev. Lett. 109, 111808 (2012) [arXiv:1205.5370].

[16] D.S. Akerib et al. [LUX Collaboration], Phys. Rev. Lett. 112, 091303 (2014) [arXiv:1310.8214].

[17] G. Cavoto, arXiv:1407.8327 [hep-ex].

[18] For recent reviews, see W. Rodejohann, J. Phys. G 39, 124008 (2012) [arXiv:1206.2560]; S.T. Petcov, Int. J. Mod. Phys. A 29, 1430028 (2014) [arXiv:1405.2263].

[19] M. Vignati, EPJ Web Conf. 70, 00044 (2014).

[20] The ATLAS and CMS Collaborations, ATLAS-CONF-2015-044 and CMS-PAS-HIG-15-002.

[21] G. Aad et al. [ATLAS Collaboration], arXiv:1406.3827 [hep-ex].

[22] T. Behnke et al., arXiv:1306.6327 [physics.acc-ph].

[23] http://clic-study.org. 